\title{
Research Paper: Relationship of Personality Traits With Quality of Life in Spouses of Patients With Physical Disabilities
}

\author{
Mahdiyeh Tavakouli Kohjehri ${ }^{1}$, ${ }^{*}$ arhad Kahrazei ${ }^{1}$
}

1- Department of Psychology, Faculty of Education and Psychology, University of Sistan and Baluchestan, Zahedan, Iran.

dtation: Tavakouli Kohjehri M, Kahrazei F. [Relationship of Personality Traits With Quality of Life in Spouses of Patients With Physical Disabilities (Persian)]. Archives of Rehabilitation. 2017; 18(2):132-141. http://dx.doi.org/10.21859/jrehab-1802130

dof: : http://dx.doi.org/10.21859/jrehab-1802130

Received: 06 Dec. 2016 Accepted: 30 Apr. 2017

Keywords: Personality traits, Quality of life, spouses of patients with physical disabilities

\section{A B STRACT}

Objective The present study was conducted with the aim to determine the relationship of personality traits with quality of life in spouses of patients with physical disabilities.

Materials \& Methods The present study was a correlational study that was conducted in 2015-2016. The study population comprised all spouses of patients with physical disabilities under the purview of Kerman. Out of them, 100 participations were selected based on availability . To evaluate the research variables, Neo five-factor personality questionnaire and 36-item form of quality of life designed by Varosherbon were used. The data of the research were analyzed using descriptive statistical and correlational and regression methods. SPSS version 21 was used for data analysis.

Results Our findings indicated that there is a positive significant correlation between personality traits of extraversion, agreeableness and conscientiousness with the quality of life among spouses of patients with physical disabilities $(\mathrm{P}<0.01)$. Also, there is a negative significant correlation between the personality trait of neurosis with the quality of life of spouses of patients with physical disabilities $(P<0.01)$. According to regression analysis findings showed that the personality traits of neurosis and extraversion predicted 17.6 and $5.2 \%$ of the variance in quality of life of spouses of patients with physical disabilities $(\mathrm{P}<0.01)$, and the personality traits of neurosis had the most contribution to the prediction of quality of life $(P<0.001)$.

Conclusion According to chronic nature of physical disabilities, participation of patients' wives in care programs can cause improvement in their quality of life and daily activities. Therefore; it is recommended to participant them in care plan through training.

\section{* Corresponding Author:}

Farhad Kahrazei, PhD

Address: Department of Psychology, Faculty of Education and Psychology, University of Sistan and Baluchestan, Zahedan, Iran.

Tel: +98 (912) 4106246

E-Mail: farhad_kahraz@ped.usb.ac.ir 


\title{
رابطه ويزَّى هاى شخصيتى با كيفيت زندگى همسران بيمار ان دهار ناتوانى جسمانى
}

\author{
مهديه توكلى كوهجرى'! "فرهاد كهرازئى'
}

ا - كروه روانشناسى، دانشكده علوم تربيتى و روانشناسى، دانشكاه سيستان و بلوجستان، زاهدان، ايران.

\begin{abstract}
حكبن
هدف يرؤهش حاضر با هدف تعيين رابطه ويرُكى هاي شخصيتى با كيفيت زندكى در همسران بيماران دجار ناتوانى جسمانى انجام شد.

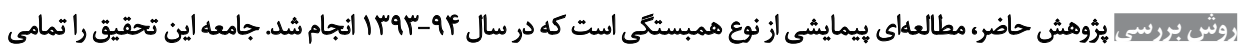

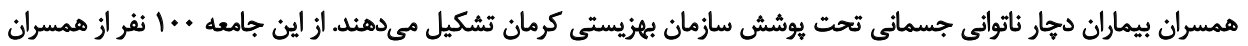

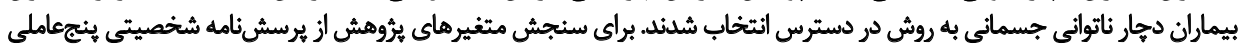

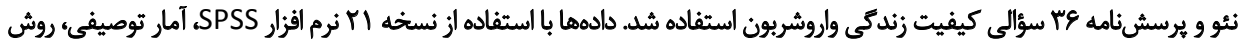

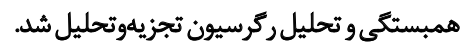

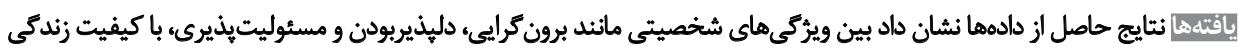

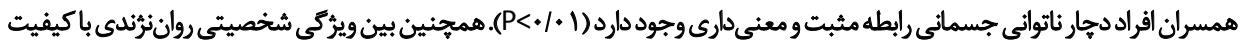

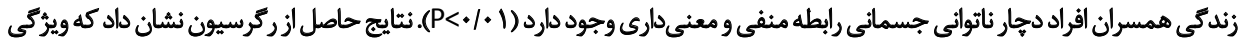

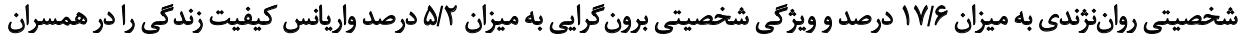

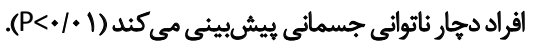

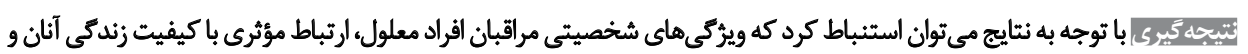

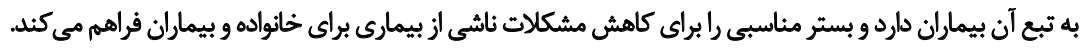

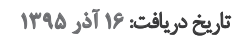

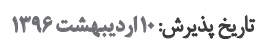

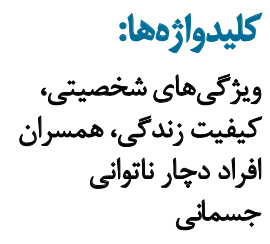

كليدوازوها:

ويرَّى هاي شخصيتي،

كيفيت زئدكى، همبران جسمانى

افراد با ناتوانىهاى حسى و جسمى تغييرات قابل توجهي

daies

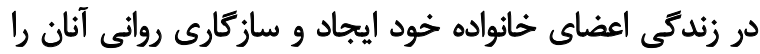

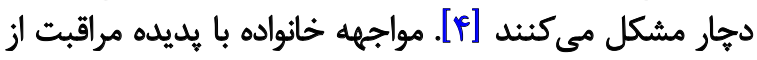

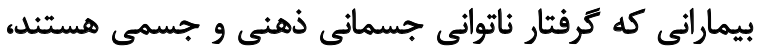

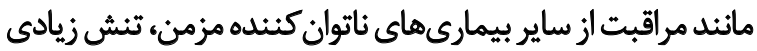

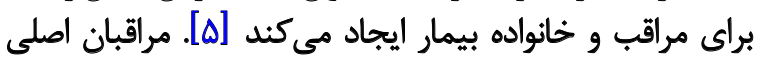

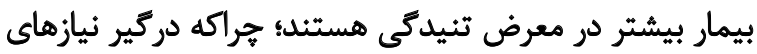

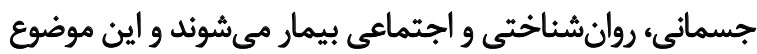

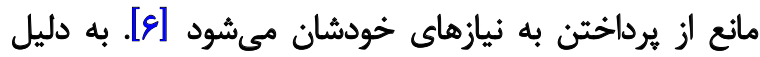

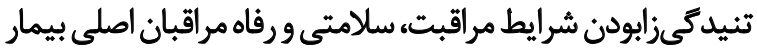

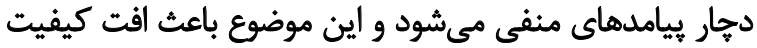

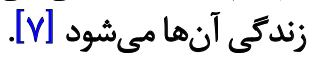

از آنجا كه همسر فرد آسيبديده مراقب اصلى و نزديكترين

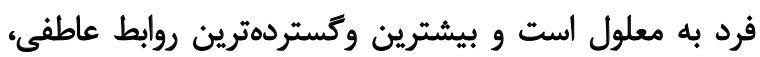
شناختى، كلامى، غيركلامى و دائم با فرد بيمار را دارد، دردين دراطئ

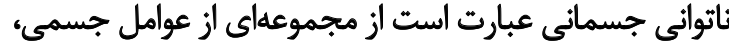

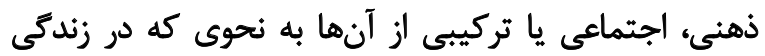

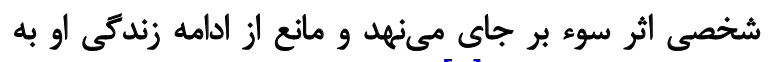

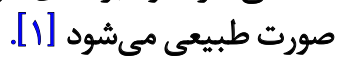
كروههاى متفاوتى از افراد وجود دارند كه به نوعى با اختلالات

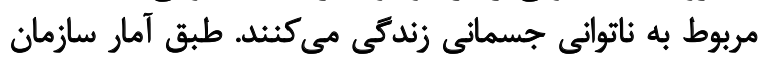

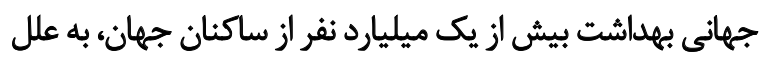

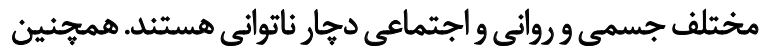

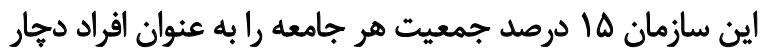

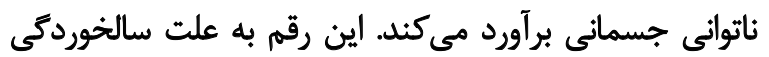

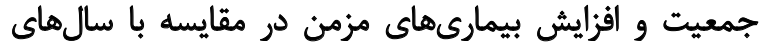

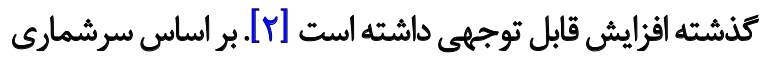

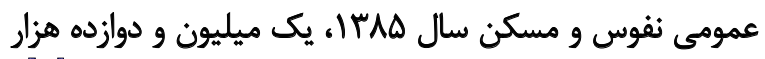

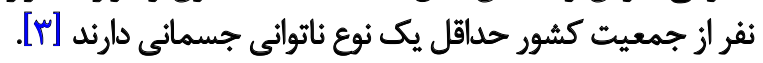

: فويسنده مسيئول: دكتر فرهاد كهرازئى

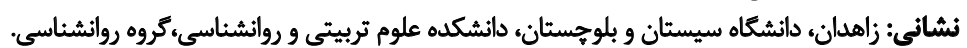
تلفن: رايانامه: farhad_kahraz@ped.usb.ac.ir 
ماستف، ترومينارس، وانهيك، هوديامونت وديورايس 'معتقدند

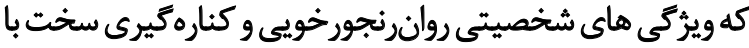

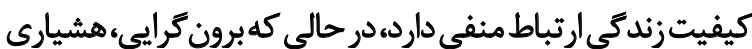

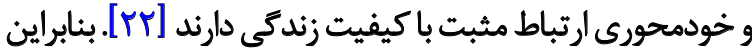

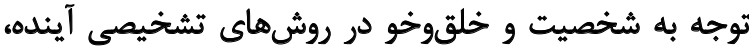

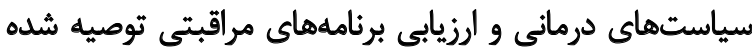

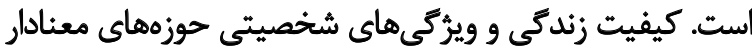

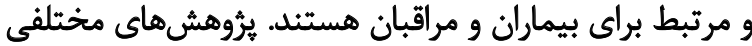

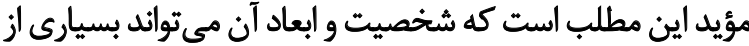

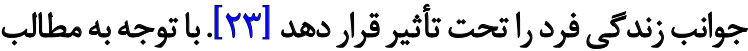

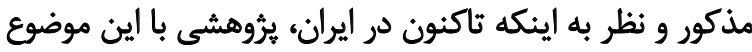

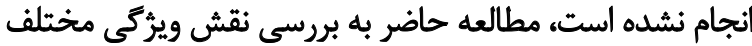

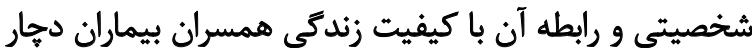

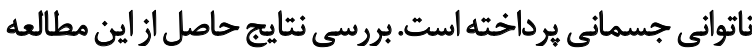

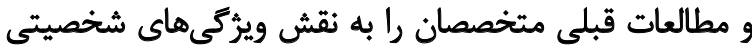

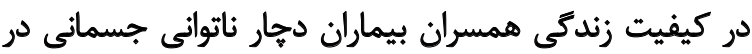

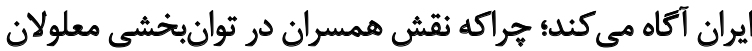

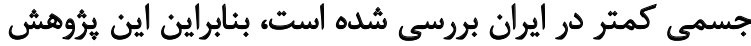

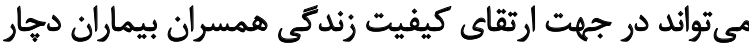

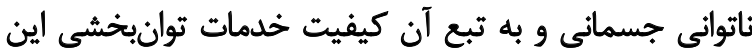

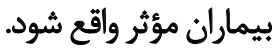

$$
\text { روش بروشى }
$$

يرؤش حاضر مطالعه بيمايشى از نوع همبستكى بود كه در

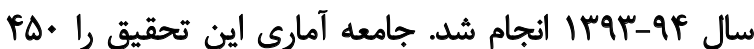

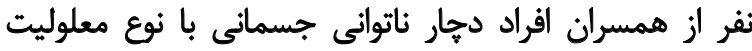

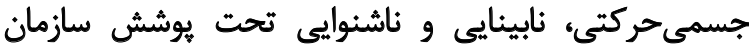

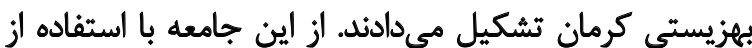

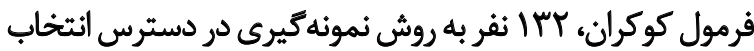

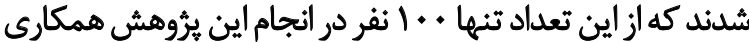

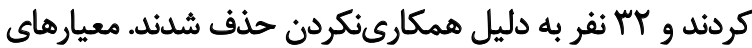

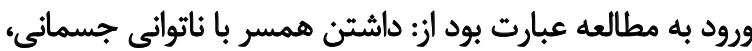

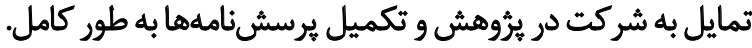

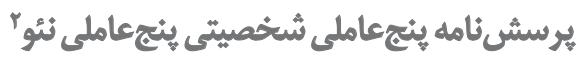

اين يرسشانامه يكى از آزمونهاى شخصيتى است كه مككرى

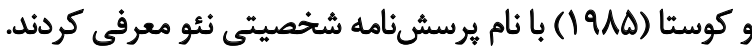

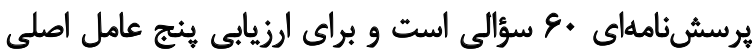

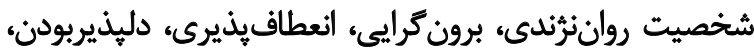

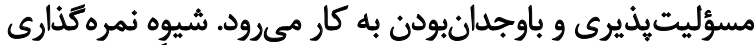
سؤالات به صورت ليكرت بئجكزينهاى شامل كاملا مخالفم (•)،

1. Masthoff, Trompenaars, Van Heck, Hodiamont, De Vries 2. NEO Five-Factor Inventory
صورت ارضانشلن نيازهاى عاطفى و ارتباط نامناسب در خانواده،

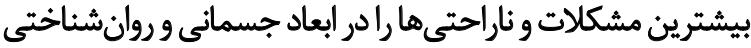

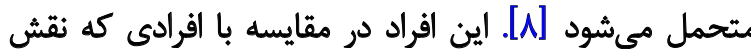

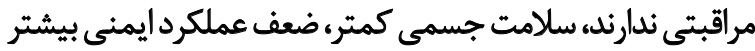

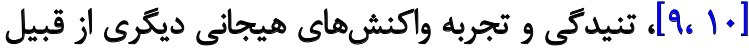

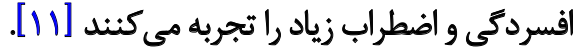

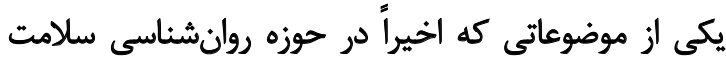

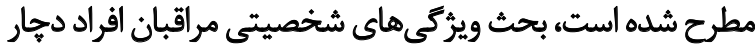

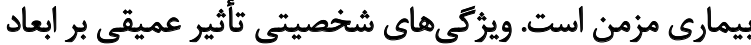

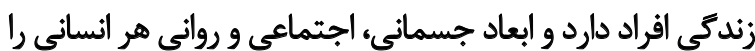

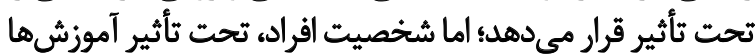

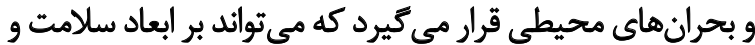

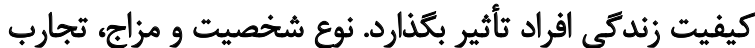

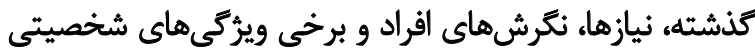

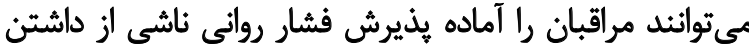

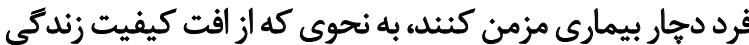

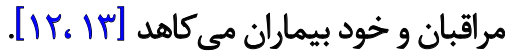
يُروهش ها نشان داده است كه ويرَّى هاى شخصيتى بر شيوه

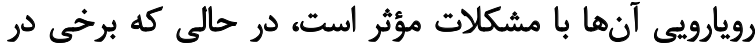

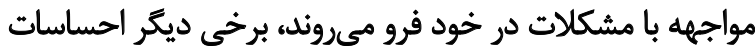

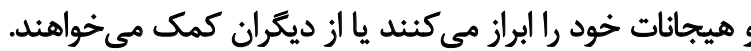

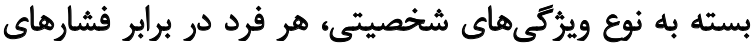

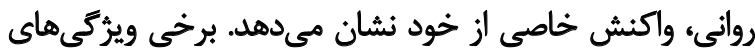

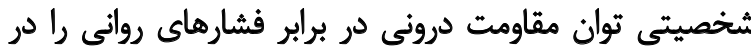

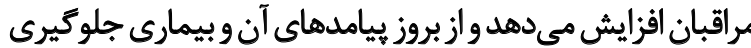

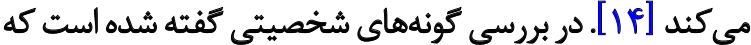

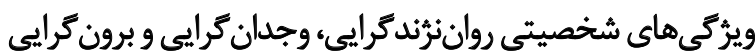

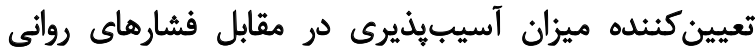

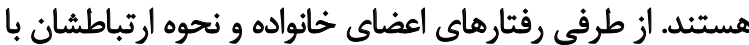

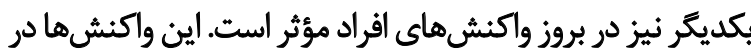

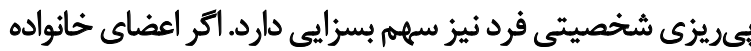

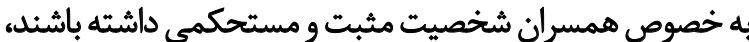

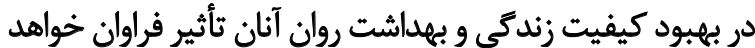

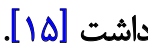

بررسىها نشان دادهاند كه زنان در انجام نقشهاي خودئ خود در

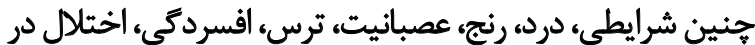

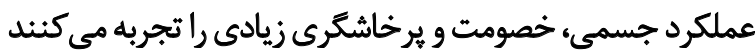

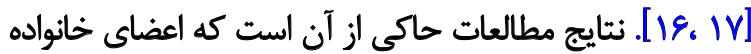

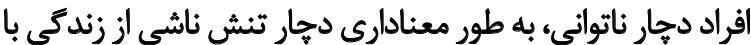

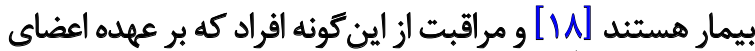

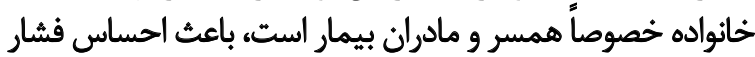

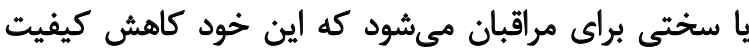

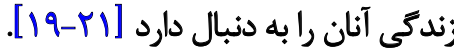




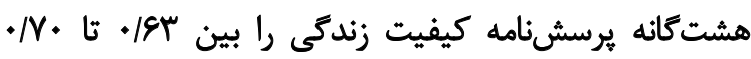

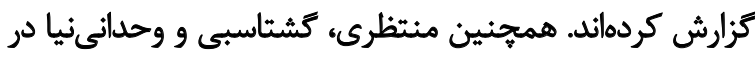
سال IFAf

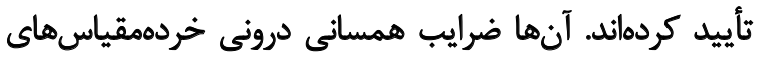

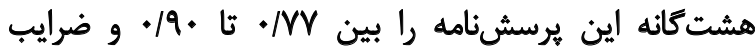

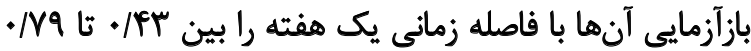

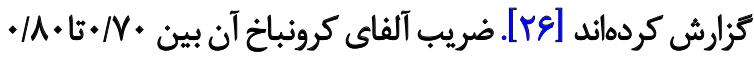

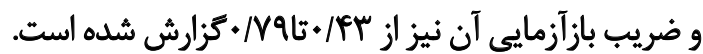

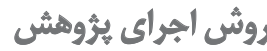

براى اجراى اين ثروهش، ابتدا هدف از اجراى تحقيق براى إي

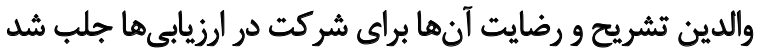

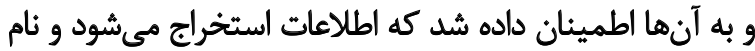

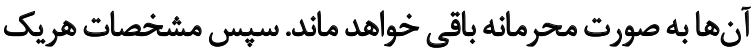

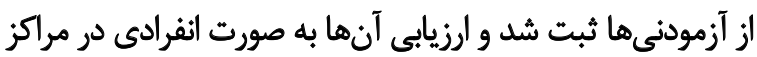

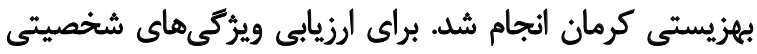

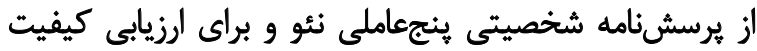

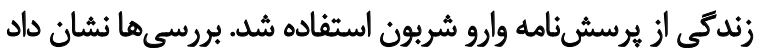

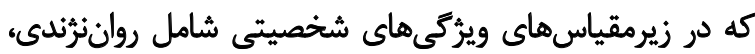

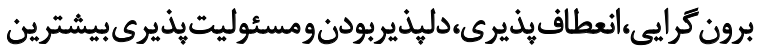

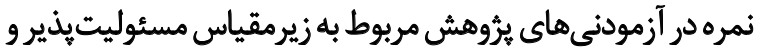

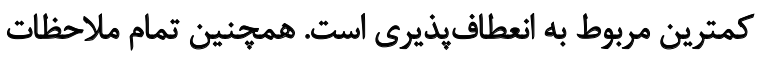

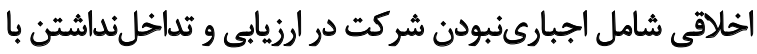

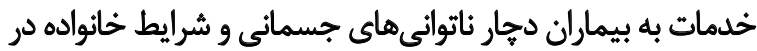

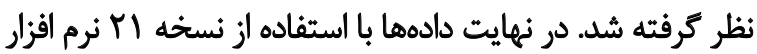
SPSS (همبستى وركرسيون) تجزيهوتحليل شد.

يافتهها

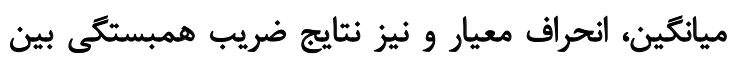

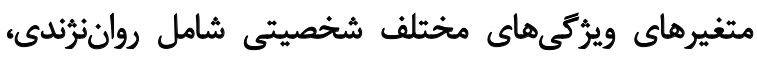

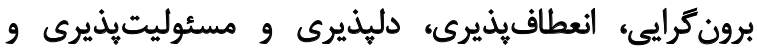

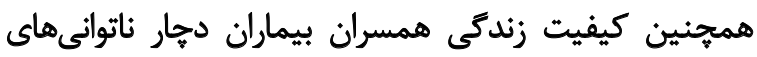

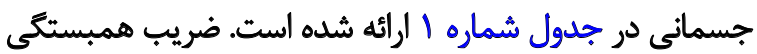

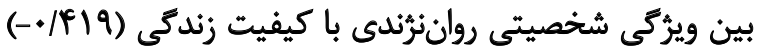

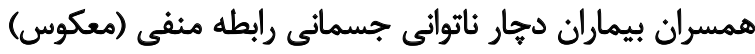

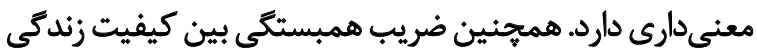

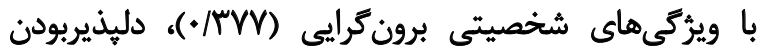

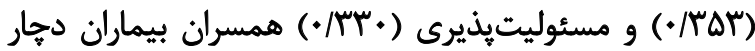

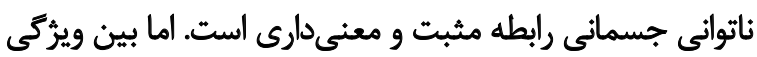

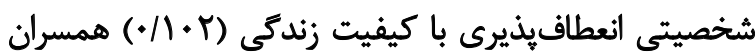

بيماران دجار ناتوانى جسمانى رابطه معنى دارى وجود ندئ زئدارد. براى تعيين وزن متغير ملاك، نمرات ويرُكىهاى شخصيتى

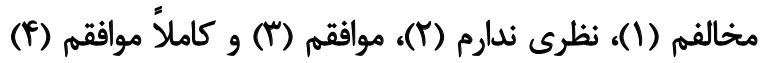

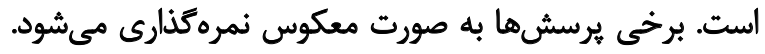

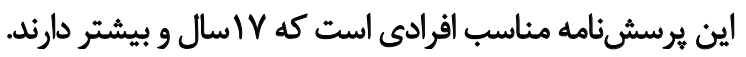

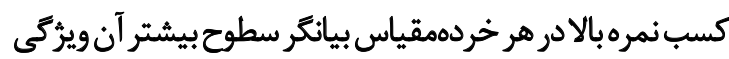

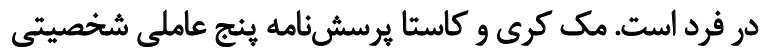

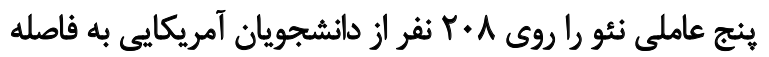

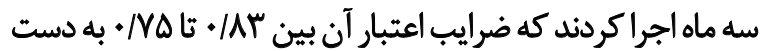

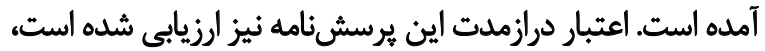

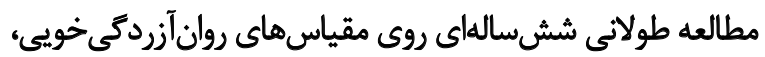

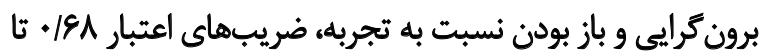

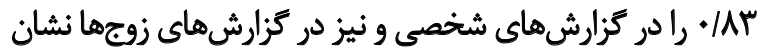

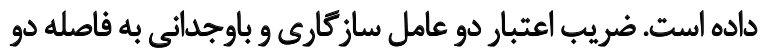

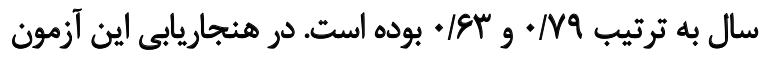

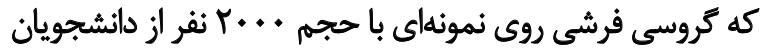

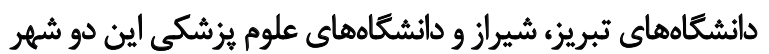

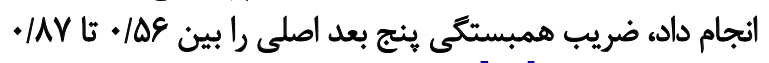

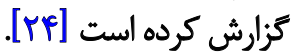

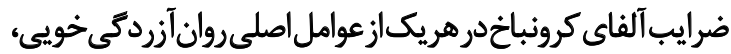

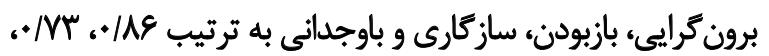

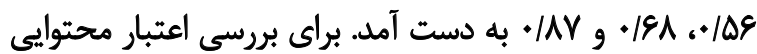

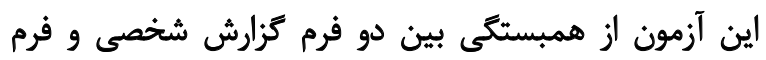

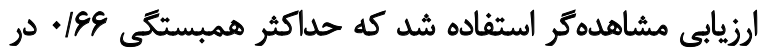

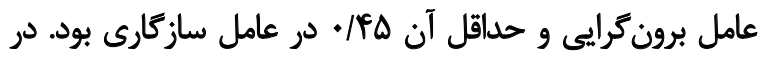

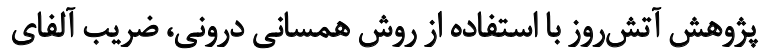

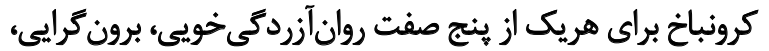

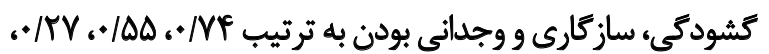

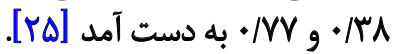

\section{برسش بامه كيفيت زئدتى (فرم كوتاه)"}

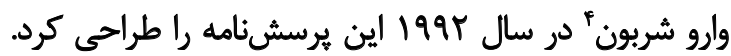

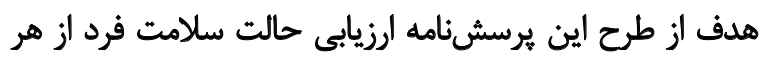

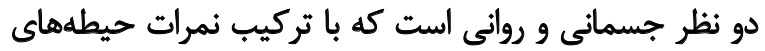

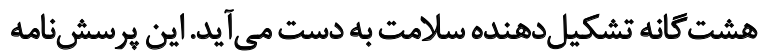

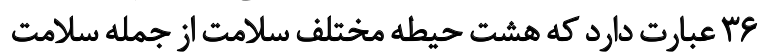

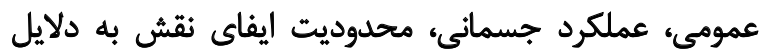

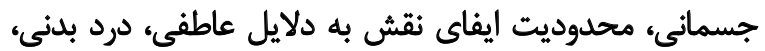

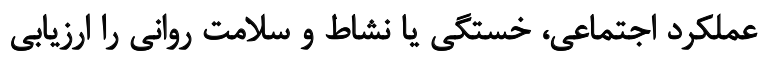

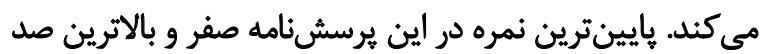

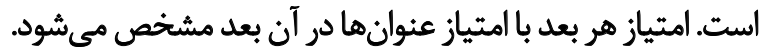

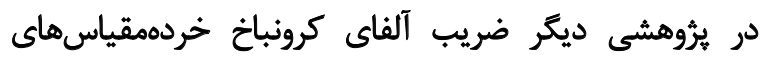

3. The Short Form Health Survey (SF-36 of Questionnaire of Life Quality

4. Ware and Sherbourne 
جدولا. ميانكين، انحراف معيار و ضريب همبستكى ويرُكىهاى شخصيتى و كيفيت زندكى همسران بيماران

\begin{tabular}{|c|c|c|c|c|}
\hline \multicolumn{2}{|c|}{ كيفيت زندكى } & \multirow{2}{*}{ اتحراف معيار } & \multirow{2}{*}{ مياتكين } & \multirow{2}{*}{ مثغيرها } \\
\hline Sig. & $\mathbf{r}$ & & & \\
\hline$+1+1$ & -.1919 & $s(\Delta)$ & $r \Delta / r^{\prime}$ & روانثزئندى \\
\hline .1 .01 &.$/ T W$ & 91.4 & $r \Delta / F r$ & برون كرايى \\
\hline.$/ N \Delta Y$ & $\cdot N \cdot r$ & $P / 9 \mid$ & $r T / \Delta V$ & انعطافيذيرى \\
\hline.$/ .1$ & . rar & $\Delta / \Lambda \Delta$ & YNIY & دليذيربودن \\
\hline \multirow[t]{2}{*}{.+.1} &.$/ \pi$ & $s / r$. & $r \cdot M$ & مسؤليتيذيزى \\
\hline & & $|V| \cdot v$ & $\Delta T / / T$ & كيفيت زندىى \\
\hline
\end{tabular}

توانبخننى

جدول Y. نتايج ركرسيون كامبه كام براى بيشبينى كيفيت زئدىى همسران أز روى ابعاد شخصيتى آنها

\begin{tabular}{|c|c|c|c|c|c|c|c|c|}
\hline Sig. & Change $\mathrm{R}^{2}$ & $R^{2}$ & $\mathbf{R}$ & $\mathbf{t}$ & $\beta$ & SD & B & الكو \\
\hline$+1 *+1$ & ./IYs &.$/ 1 v 9$ &.$/ 119$ & $-r / r q$ &.$- / \pi$ &.$/ T \Delta \Delta$ &.$- / A r \Lambda$ & روانثرثندى \\
\hline$\% / * 1 r$ & $.0 \Delta r$ & ג &.$/ F r \Lambda$ & $r / \Delta F$ & . & . /TYs & $\cdot N \cdot F$ & برون كرايى \\
\hline
\end{tabular}

ويرُكى هاى شخصيتى رواننرُندى با كيفيت زندكى همسران

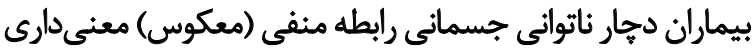

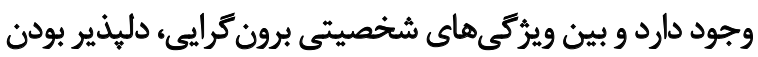

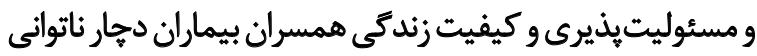

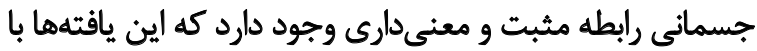
يثروهش هاى ذكر شده همسو و همخوان است نقش حمايتهاى اجتماعى از جانب خانواده در سازئارى

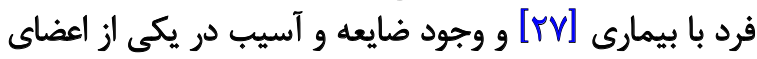

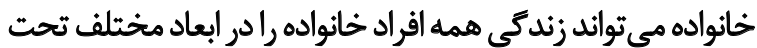

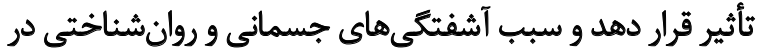

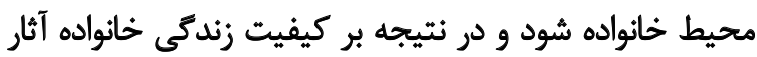

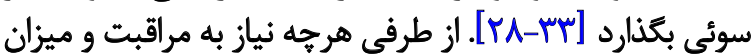

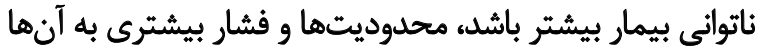

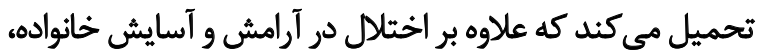

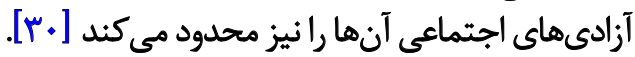
با توجه به مسئوليتيذيرىهاى جديد و تغييرات نقش،

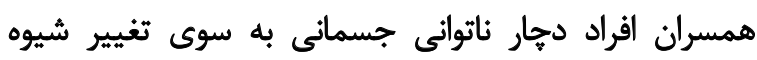

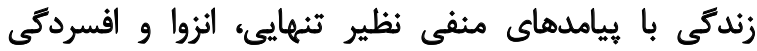

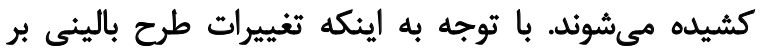

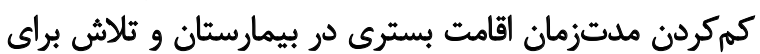

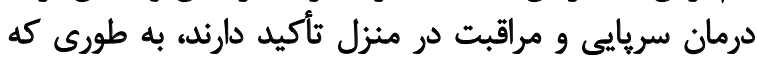

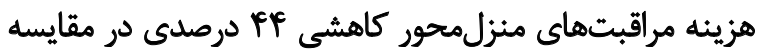

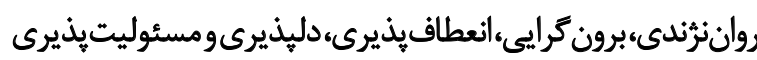

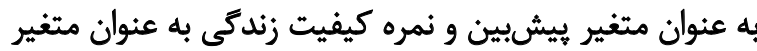

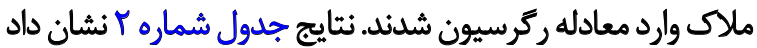

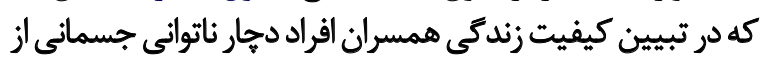

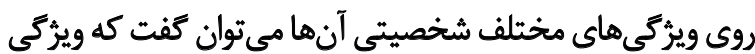

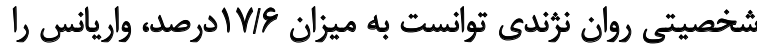

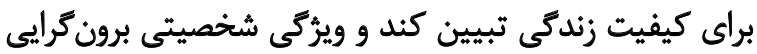

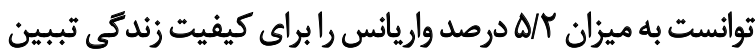

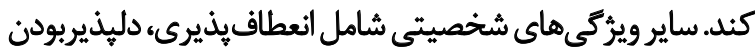

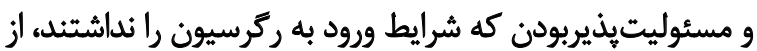
معادله حذف شدئد.

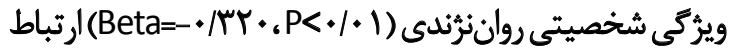

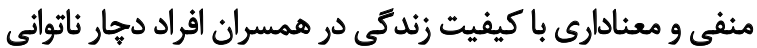

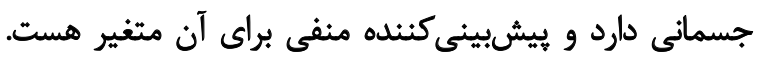

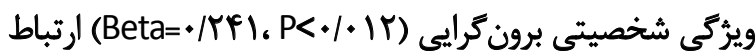

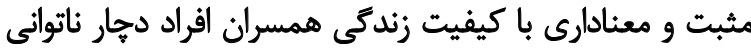

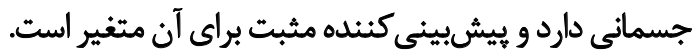
$\Leftrightarrow$

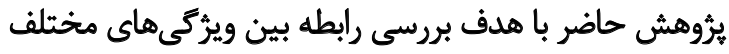

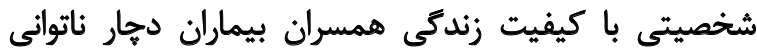

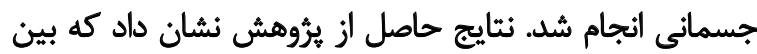


اختلالات روانى دجار شدهاند كه مجموع اين عوامل تأثير بسيارى

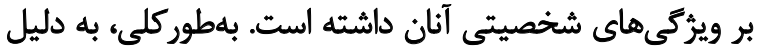

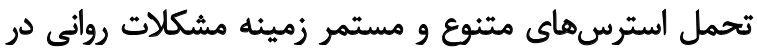

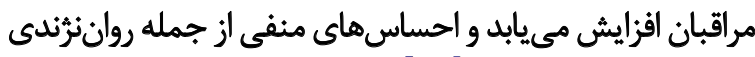

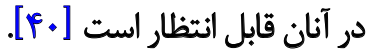

در همسران بعضى صفات شخصيتى مانتد روان نرثندى مستقيماً

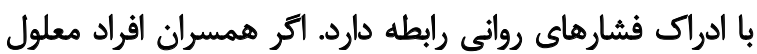

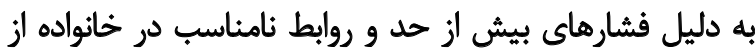

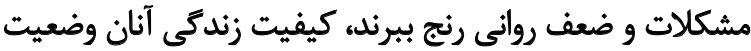

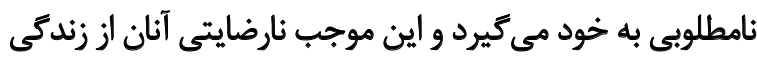

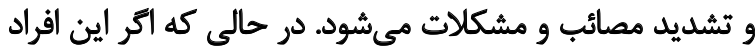

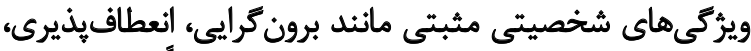

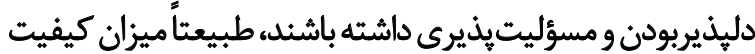

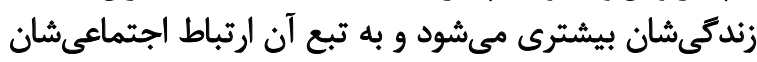

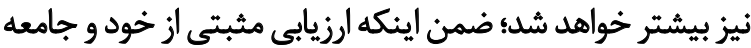

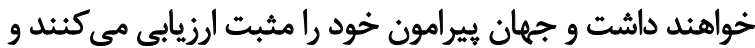

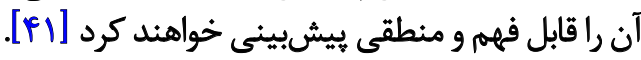

\section{نتيجهيرى}

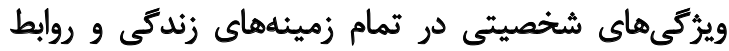

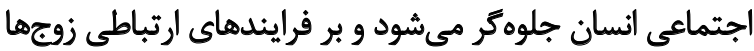

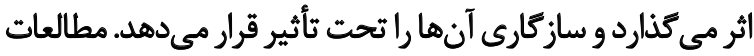

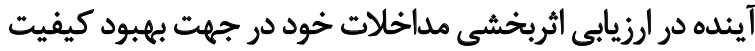

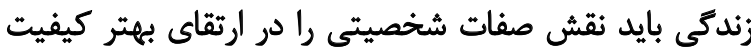

$$
\text { زندكى در نظر بكيرند. }
$$

اين يُروهش با محدوديتهاى بسيارى از جمله دسترسى آسان

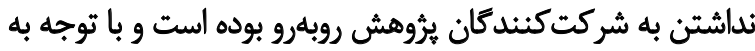

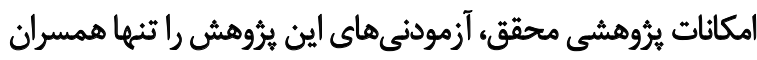

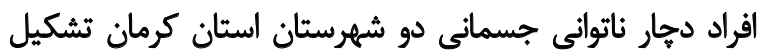

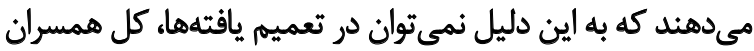

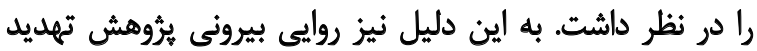

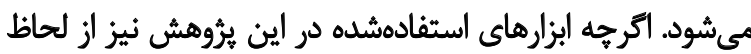

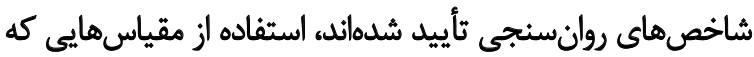

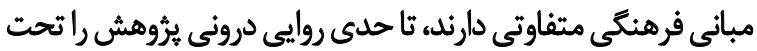

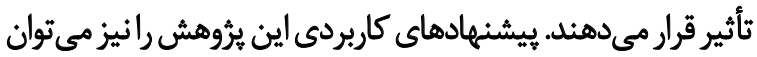

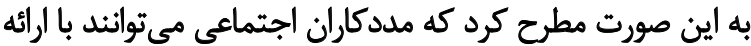

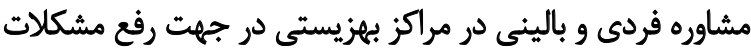

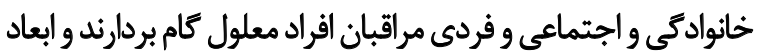

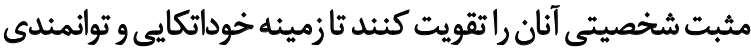

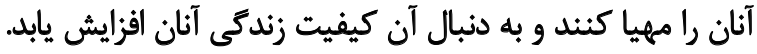

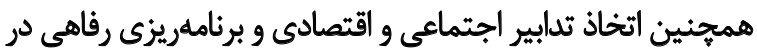

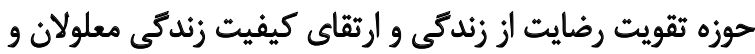

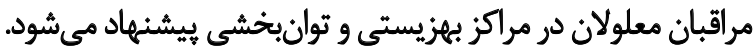

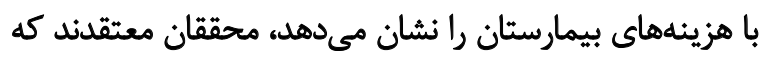

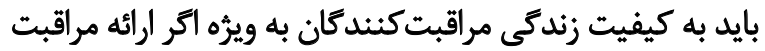

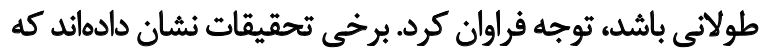

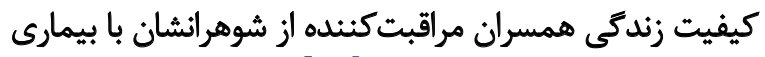

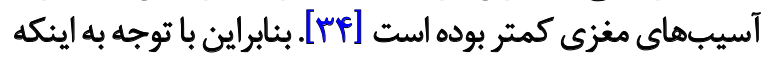

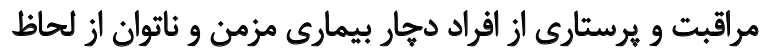

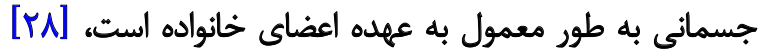

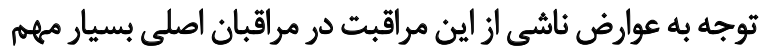

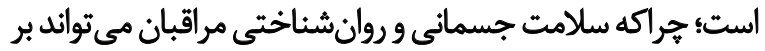

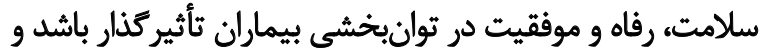

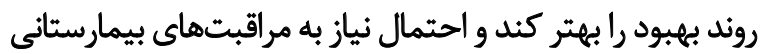

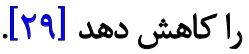

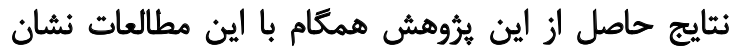

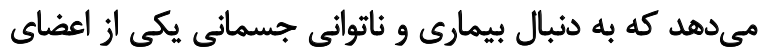

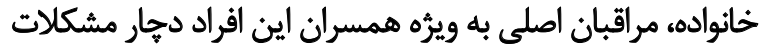

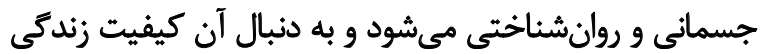

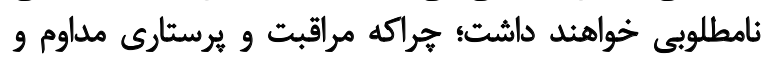

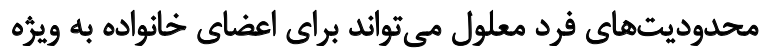

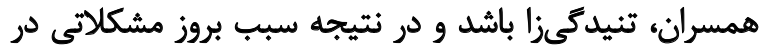

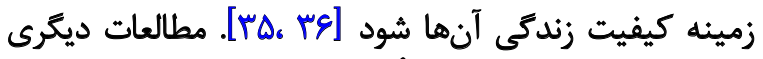

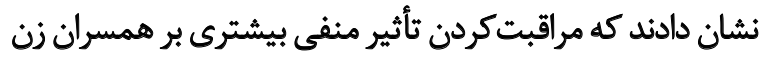

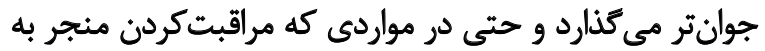

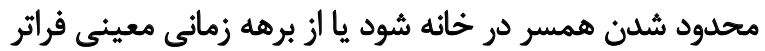

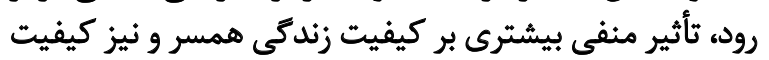

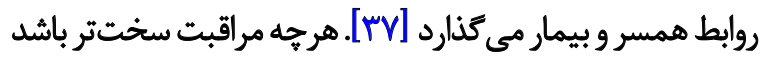

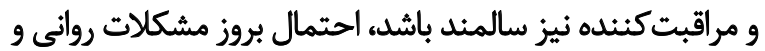

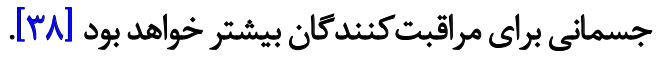
در زمينه بررسى عوامل ثأثير كذار در سلامت و بهبيود زندئى

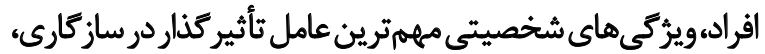

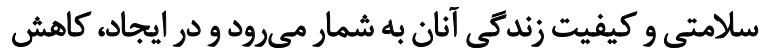

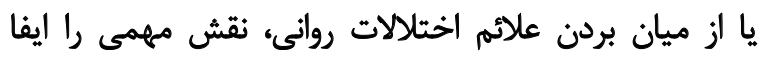

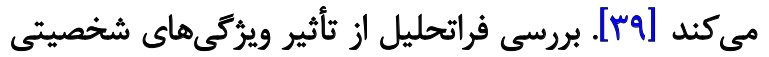

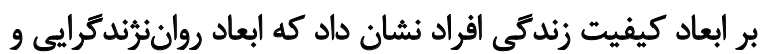

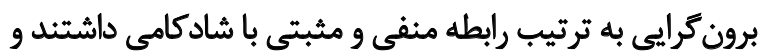

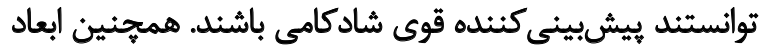

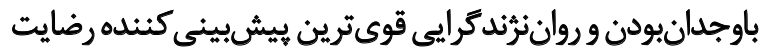

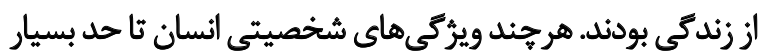

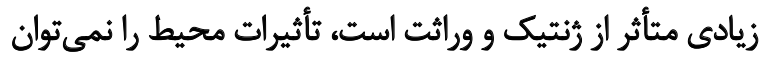

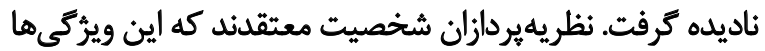

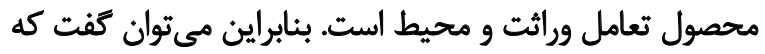

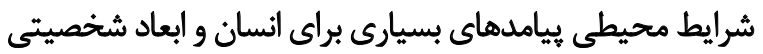

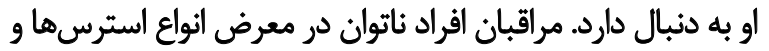

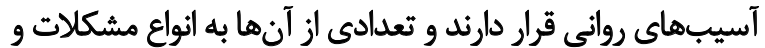


در هايان، انجام اين يُروهش از اين لحاظ اهميت دارد كه نشان

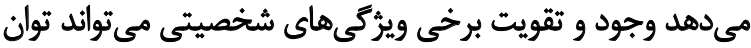

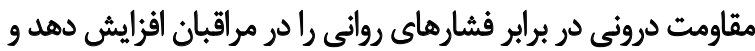

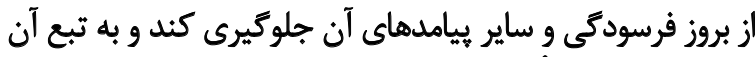

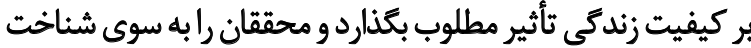

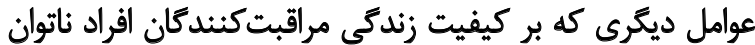
تأثير مي كذارد، سوق دهائ كه بر كيفيت

با توجه به اينكه اين ثرؤهش روى همسران بيماران دجار

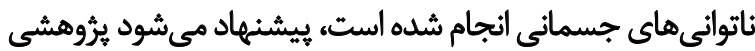

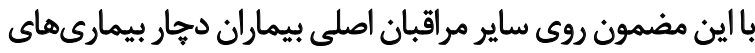

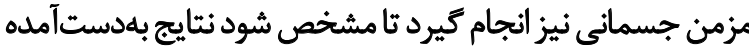

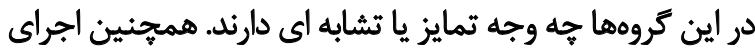

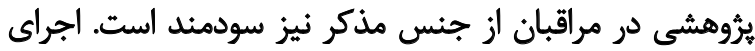

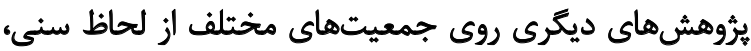

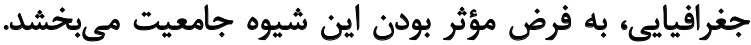

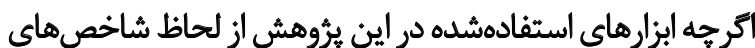

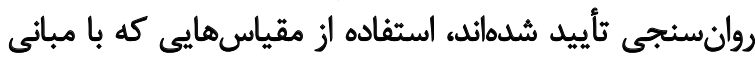

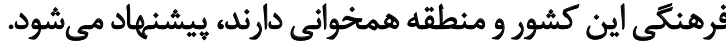

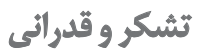

بدين وسيله از مسئولان محترم سازمان بهزيستى استان كرمان

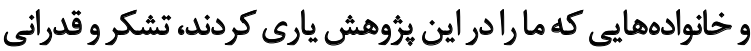

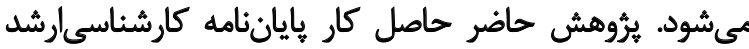

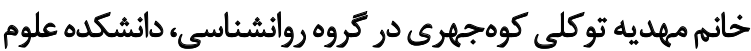

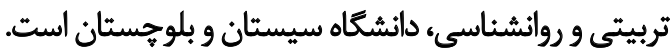




\section{References}

[1] Pourhossein Hendabad P, Eghlima M, Arshi M, Taghinezhad Z. [Research Paper: Effects of social skills training on social participation among physical and motor disabled people in educational complex charity, Raad Center (Persian)]. Archives of Rehabilitation. 2016 17(4):308-17. doi: 10.21859/jrehab-1704308

[2] World Health Organization. World report on disability. Geneva: World Health Organization; 2011

[3] Sajjadi H, Zanjari N. [Disability in Iran: Prevalence, characteristics, and socio-economic correlates (Persian)]. Archives of Rehabilitation. 2015; 16(1):36-47.

[4] Jeon B, Kwon S, Kim H. Health care utilization by people with disabilities: A longitudinal analysis of the Korea Welfare Panel Study (KoWePS). Disability and Health Journal. 2015; 8(3):353-62. doi: 10.1016/j.dhjo.2015.01.001

[5] Mahdinejad L, Goharpey S, Ghasem Zadeh R, Latifi SM, Bakhshande Bavarsad M, Mansouri N. [A comparison of family functioning in families with member visually and hearing impairment with families without disorder (Persian)]. Journal of Modern Rehabilitation. 2016; $9(6): 42-50$.

[6] Jorstad ML. Perceived life changes and feelings of depression in caregivers of persons with stroke [MSc. thesis]. Toledo: University of Toledo; 2004

[7] Farhadi A, Foroughan M, Mohammadi F, Rassouli M, Sadegh Moghadam L, Nazari S, et al. [Caregiving appraisal in family caregivers of older adults (Persian)]. Iranian Journal of Ageing. 2016; 11(1):8-19. doi: 10.21859 /sija-110108

[8] Soleimani M, Azimian S, Nazari AM, Zahrakar K. [Relationship between family power structure, marital conflicts, and mental health in male retirees of Alborz province department, Iran ministry of education (Persian)]. Iranian Journal of Ageing. 2016; 10(4):80-87.

[9] Dalvandi A, Heikkilä K, Maddah SSB, Khankeh HR, Ekman SL. Life experiences after stroke among Iranian stroke survivors. International Nursing Review. 2010; 57(2):247-53. doi: 10.1111/j.14667657.2009.00786.x

[10] Hélène Ezer, Nicole Ricard, Louise Bouchard, Souhami L, Saad F, Aprikian A, et al. Adaptation of wives to prostate cancer following diagnosis and 3 months after treatment: A test of family adaptation theory. International Journal of Nursing Studies. 2006; 43(7):827-38. doi: 10.1016/j.ijnurstu.2006.05.004

[11] Nazer M, Riyahi N, Mokhtaree M. [The effect of stress management training with cognitive behavioral style on stress and mental health of parents of children with intellectual disabilities (Persian)]. Archives of Rehabilitation. 2016; 17(1):32-41.

[12] Chen L, Liu J, Zhang J, Lu X. Non-pharmacological interventions for caregivers of patients with schizophrenia: A meta-analysis. Psychiatry Research. 2016; 235:123-7. doi: 10.1016/j.psychres.2015.11.037

[13] Lin RF, Chang JJ, Lu YM, Huang MH, Lue YJ. Correlations between quality of life and psychological factors in patients with chronic neck pain. The Kaohsiung Journal of Medical Sciences. 2010; 26(1):13-20. doi: 10.1016/s1607-551x(10)70003-6

[14] Rajabi Damavandi G, Poushaneh K, Ghobari Bonab B. [Relationship between personality traits and coping strategies in parents of children with autistic spectrum disorders (Persian)]. Journal of Exceptional Children. 2010; 9(2):133-145

[15] Sharafi AS. [The relationship between religious orientation, family communication patterns, mental health and and personality traits of students (Persian)]. Paper presented at: The First National Conference of Personality and Modern Life; 2013 May 23-24; Sanandaj, Iran.

[16] Shanfield SB. Myocardial infarction and patients' wives. Psychosomatics. 1990; 31(2):138-45. doi: 10.1016/s0033-3182(90)72186-x

[17] Coulombe S, Jutras S, Labbé D, Jutras D. Residential experience of people with disabilities: A positive psychology perspective. Journal of Environmental Psychology. 2016; 46:42-54. doi: 10.1016/j.jenvp.2016.03.002

[18] Iezzoni LI, Wint AJ, Kuhlthau KA, Boudreau AA. Adults' recollections and perceptions of childhood caregiving to a parent with significant physical disability. Disability and Health Journal. 2016; 9(2):208-217. doi: 10.1016/j.dhjo.2015.10.009

[19] Ring A, Jacoby A, Baker GA, Marson A, Whitehead MM. Does the concept of resilience contribute to understanding good quality of life in the context of epilepsy? Epilepsy \& Behavior. 2016; 56:153-64 doi: 10.1016/j.yebeh.2016.01.002

[20] Grant G, Ramcharan P, Goward P. Resilience, family care, and people with intellectual disabilities. International Review of $\mathrm{Re}$ search in Mental Retardation. 2003; 135-73. doi: 10.1016/s0074 7750(03)01004-8

[21] Dalvand H, Hosseini SA, Dehghan L, Feizi A. [Investigation of quality of life in mothers of children with spinabifida based on children's hoffer ambulatory criteria (Persian)]. Archives of Rehabilitation. 2015; 16(2):148-155

[22] Masthoff ED, Trompenaars FJ, Van Heck GL, Hodiamont PP, De Vries J. The relationship between dimensional personality models and quality of life in psychiatric outpatients. Psychiatry Research. 2007; 149(1-3):81-8. doi: 10.1016/j.psychres.2006.01.004

[23] Nezamipour E, Ahadi H. [The effect of cognitive emotion regulation strategies on the relationship between personality traits and quality of life in dialysis patients (Persian)]. Qom University of Medical Sciences Journal. 2016; 10(2):70-80

[24] Rena F, Moshe S, Abraham O. Couples' adjustment to one partner's disability: The relationship between sense of coherence and adjustment. Social Science \& Medicine, 1996; 43(2):163-171. doi: 10.1016/0277-9536(95)00358-4

[25] Yu J, Tang YY. Reliability, validity, and responsiveness of the short form 36 (SF-36) health survey questionnaire in patients with Graves' disease. Asian Biomedicine. 2013; 7(3):391-397. doi: 10.5372/1905 7415.0703 .191

[26] Montazeri A, Ghastasbi A, Vahdani Niya M. [Translation and determination of validity and stability of Persian SF-36 Questionnaire (Persian)]. Payesh. 2005; 5(1):49-56 .

[27] Rahmani Rasa A, Hosseini SA, Haghgoo H, Khankeh HR, Ray GG [Review paper: Aspects related to resilience in people with spinal cord injury (Persian)]. Archives of Rehabilitation. 2017; 17(4):350-359.

[28] Given CW, Given B, Stommel M, Collins C, King S, Franklin S. The Caregiver Reaction Assessment (CRA) for caregivers to persons with 
chronic physical and mental impairments. Research in Nursing \& Health. 1992; 15(4):271-83. doi: 10.1002/nur.4770150406

[29] Berg-Weger M, Rubio DM, Tebb S. Strengths-based practice with family caregivers of the chronically Ill: Qualitative insights. Families in Society: Journal of Contemporary Social Services. 2001; 82(3):263-72. doi: 10.1606/1044-3894.191

[30] Rahmani Anaraki H, Mahmoudi Gholam R, Roohi G, Asayesh H, Nasiri H, Rakhshani H. [Genral health status of neurologic patients' caregivers and the related factors (Persian)]. Journal of Research Development in Nursing \& Midwifery. 2013; 9(2):49-55.

[31] Sharifi M, Fatehizade M. [Correlation between religious coping with deppression and caregiver burnout in family caregivers (Persian)]. Modern Care Journal. 2012; 9(4):327-335.

[32] Vollrath M, Torgersen S. Personality types and coping. Personality and Individual Differences. 2000; 29(2):367-78. doi: 10.1016/s01918869(99)00199-3

[33] Koren ME, Czurylo K, Epsom R, Gattuso M, Stark B, Zastrow P, et al. Nurses' work environment and spirituality: A descriptive study. International Journal of Caring Sciences. 2009; 2(3):118-125.

[34] Carlozzi NE, Kratz AL, Sander AM, Chiaravalloti ND, Brickell TA, Lange RT, et al. Health-related quality of life in caregivers of individuals with traumatic brain injury: Development of a conceptual model. Archives of Physical Medicine and Rehabilitation. 2015; 96(1):105-13. doi: 10.1016/j.apmr.2014.08.021

[35] Weitzenkamp DA, Gerhart KA, Charlifue SW, Whiteneck GG, Savic G. Spouses of spinal cord injury survivors: The added impact of caregiving. Archives of Physical Medicine and Rehabilitation. 1997; 78(8):822-7. doi: 10.1016/s0003-9993(97)90194-5

[36] Isa SN, Aziz AA, Rahman AA, Ibrahim MI, Ibrahim WP, Mohamad $\mathrm{N}$, et al. The impact of disabled children on parent health-related quality of life and family functioning in kelantan and its associated factors. 2013; 34(4):262-8. doi: 10.1097/DBP.0b013e318287cdfe

[37] Nolan R, Luther B, Young P, Murphy NA. Differing perceptions regarding quality of life and inpatient treatment goals for children with severe disabilities. Academic Pediatrics. 2014; 14(6):574-80. doi: 10.1016/j.acap.2014.02.012

[38] Jafari F, Moien L, Soroush MR, Mosavi B. [Quality of life in chemical warfare victims with ophthalmic damage's spouses (Persian)]. Iranian Journal of War and Public Health. 2007; 3(11):8-12.

[39] Kentros MK, Terkelsen K, Hull J, Smith TE, Goodman M. The relationship between personality and quality of life in persons with schizoaffective disorder and schizophrenia. Quality of Life Research. 1997; 6(2):118-22. PMID: 9161111

[40] Martens L, Addington J. The psychological well-being of family members of individuals with schizophrenia. Social Psychiatry and Psychiatric Epidemiology. 2001; 36(3):128-33. doi: 10.1007/ s001270050301

[41] Moody, LE, McMillan S. Dyspnea and quality of life indicators in hospice patients and their caregivers. Health and Quality of Life Outcomes. 2003; 1(1):9. doi: 10.1186/1477-7525-1-9 
